\title{
The accuracy of MUST assessments by staff in a care home setting
}

\author{
H. E. Travis and M. Mackenzie-Fraser \\ Robert Gordon University, St. Andrews Street, Aberdeen AB25 1HG, UK
}

\begin{abstract}
Malnutrition is an area of care that can go neglected, leading to health complications and a decreased quality of life. The Nutrition Screening Survey has found that $52 \%$ of hospital admissions from care homes are affected by malnutrition. The Malnutrition Universal Screening Tool (MUST) is widely used to screen for malnutrition in both primary and secondary care, but there is little information on its use in a community setting,

The aim of this research was to investigate the accuracy of MUST when used in care homes and to identify sources of error. The investigator independently calculated MUST scores for 148 care home residents across 6 care homes within the same city using their most recently recorded height and weight. Each step of the scores was compared to the corresponding score calculated by care home staff. The action that was taken in response to MUST scores was noted and compared to the recommended action according to MUST guidelines. Moderate agreement was found between the MUST scores calculated by care home staff compared to those calculated by the investigator using the Kappa measure of agreement $(k=0.687)$. A particular area of error was found in the calculation of percentage weight loss as less than moderate agreement was found between the scores from care home staff compared to the investigator $(k=0.385)$. Whether the correct management was put into place was found to be significantly dependent on the care home that carried out the screening and on the individual's risk of malnutrition. However, it was found to be significantly independent of which care home had carried out the screening. Despite this, the percentage of residents who had the correct action put in place for them at each care home is still worth considering.
\end{abstract}

\begin{tabular}{lc}
\hline Care Home & \% of Correct Action Taken \\
\hline Care Home 1 & 88.9 \\
Care Home 2 & 82.6 \\
Care Home 3 & 91.3 \\
Care Home 4 & 86.4 \\
Care Home 5 & 84.2 \\
Care Home 6 & 75.0 \\
\hline
\end{tabular}

Recommendations include further research to investigate the training that is given to care home staff in regards to MUST. A review of the documentation used to record MUST scores and the guidelines used for management of malnutrition may be beneficial, and the inclusion of a column to allow staff to record the calculated percentage weight loss would be useful to encourage staff to calculate it correctly and to provide further insight into the extent of any errors. 